\title{
Gamma-ray luminosity properties of gamma-ray pulsars
}

\author{
L. Zhang ${ }^{1,2}$, Z. X. Han ${ }^{2}$, and Z. J. Jiang ${ }^{2}$ \\ ${ }^{1}$ National Astronomical Observatories/Yunnan Observatory, Chinese Academy of Sciences, PO Box 110, \\ Kunming, PRC, PR China \\ e-mail: astroynu@public.km.yn.cn \\ ${ }^{2}$ Department of Physics, Yunnan University, Kunming, PR China
}

Received 2 June 2004 / Accepted 30 August 2004

\begin{abstract}
Based on a new self-consistent outer gap model, we statistically study luminosity properties of $\gamma$-ray pulsars with ages of $\leq 2 \times 10^{7} \mathrm{yr}$ in the Galaxy as well as those with ages of $\leq 5 \times 10^{6} \mathrm{yr}$ in the Gould belt using a Monte Carlo method, where the $\gamma$-ray beaming effect has been included. Generally, the relation between $\gamma$-ray luminosity, $L_{\gamma}$, and pulsar spin-down power, $L_{\mathrm{sd}}$, can be expressed as $L_{\gamma} \propto L_{\mathrm{sd}}^{\delta}$, where $\delta$ is a parameter that depends on the galactic latitude and the $\gamma$-ray threshold. For a given $\gamma$-ray threshold (for example, the EGRET threshold or GLAST threshold), the parameter $\delta$ is an increasing function of the galactic latitude. For the EGRET threshold, $\delta$ changes smoothly with the galactic latitude. For the GLAST threshold, however, $\delta$ has a minimum at $|b| \leq 5^{\circ}$, and $\delta$ increases by a factor of $\sim 2$ at the range of $|b|=5^{\circ}-10^{\circ}$ relative to $\delta$ at $|b| \leq 5^{\circ}$, and then increases smoothly as the galactic latitude further increases. The reason for this is that high galactic latitude $\gamma$-ray pulsars are dominated by mature pulsars. In fact, the ratio of $\gamma$-ray pulsars with their ages of $>10^{6} \mathrm{yr}$ to all simulated $\gamma$-ray pulsars also increases with the galactic latitude. For the $\gamma$-ray pulsars produced in a fixed range of galactic latitude, the parameter $\delta$ increases slightly with the sensitivity of $\gamma$-ray detector.
\end{abstract}

Key words. gamma-rays: theory - stars: pulsars: general - stars: neutron - stars: statistics

\section{Introduction}

The Energetic Gamma Ray Telescope (EGRET) has found $271 \gamma$-ray point sources listed in the third EGRET (3EG) cata$\log$, which 170 have not been identified (Hartman et al. 1999). The nature of these unidentified EGRET sources has been widely studied, including their space distribution, spectral features, variability and possible counterparts. It is generally believed that most unidentified EGRET sources at the Galactic plane $\left(|b|<5^{\circ}\right)$ correlate with the Galactic objects such as OB associations, supernova remnants (SNRs), young pulsars, HII regions and young open clusters (for example, Montmerle 1979; Halpern \& Ruderman 1993; Helfand 1994; Kaaret \& Cottam 1996; Yadigaroglu \& Romani 1997; Zhang \& Cheng 1998; Romero et al. 1999; Zhang et al. 2000; Bhattacharya et al. 2003). Recently, several unidentified EGRET sources have been found to be positionally coincident with the newly discovered pulsars in the released portion of the Parkes multibeam radio survey (Camilo et al. 2000; Torres et al. 2001), Kramer et al. (2003) found that there are 37-38 positional coincidences between the unidentified EGRET sources and the observed radio pulsars and 19 plausible associations. In the midlatitude region, Gehrels et al. (2000) singled out a population of steady unidentified EGRET sources; the spectral properties of these sources are significantly softer, fainter and have a steeper $\log N-\log S$ function than those at low latitudes (Gehrels et al. 2000). Using a different selection standard, Grenier (2000) obtained a population of persistent unidentified EGRET sources.

The steady or persistent unidentified EGRET sources are correlated with the tilted Gould belt and about 40 of them have associated with the Gould belt at $|b|>5^{\circ}$ (Grenier 2000). These sources have been suggested to associate with recent supernovae in the nearby Gould belt (Grenier 1997; Gehrels et al. 2000; Grenier 2000; Grenier 2003). Therefore, a possibility is that at least part of the steady or persistent unidentified EGRET sources are the pulsars which emit $\gamma$-rays in the Gould belt. Based on the polar cap models, Harding \& Zhang (2001) suggested that the off-beam $\gamma$-rays come from high-altitude curvature emission of primary particles and can radiate over a large solid angle and have a much softer spectrum than those of the main beams, and at least some of radio-quiet Gould belt sources detected by EGRET could be such off-beam $\gamma$-ray pulsars. In the framework of the outer gap model, Cheng et al. (2004) investigated the emission properties of $\gamma$-ray pulsars in Galaxy and in the Gould belt.

In this paper, we study $\gamma$-ray luminosity properties of $\gamma$-ray pulsars in the Galaxy as well as in the Gould belt, based on the outer gap model of $\gamma$-ray emission from the pulsars (Zhang et al. 2004). 


\section{2. $\gamma$-ray luminosity of $\gamma$-ray pulsars}

We use the outer gap model of Zhang et al. (2004) to estimate the $\gamma$-ray luminosity of each pulsar. Compared to previous outer gap models (Cheng et al. 1986a,b; Zhang \& Cheng 1997), three important effects in this revised outer gap model are included: (i) the effect of the inclination angle $(\alpha)$, which is the angle between the magnetic axis and the rotation axis, in determining the size of the outer gap; (ii) the fact that the typical radiation region of the outer gap is not necessary at half of the light cylinder; instead it should be better represented by an appropriate average over the entire outer gap. This effect is particularly important for old pulsars. When the gap size in this region is larger than unity, the outer gap is assumed to be turned off; and (iii) as long as the gap size is less than unity in some parts of the magnetosphere, the outer gap still exists. This effect allows some pulsars with an appropriate combination of $\alpha, P$ and $B$, to maintain their outer gaps until they are a few million years old. These pulsars are able to move up to high galactic latitude and their ages make them weak $\gamma$-ray sources.

An outer gap can start at the null charge surface $(\boldsymbol{\Omega} \cdot \boldsymbol{B}=0)$ of the pulsar magnetosphere (it should be noted that Horitani \& Shibata (2002) assumed that the outer gap can start either below or above the null charge surface), which defines the inner boundary of the outer gap and the radial distance is $r_{\text {in }}$. After considering the effect of the magnetic inclination angle and magnetic geometry, Zhang et al. (2004) modified the outer gap model proposed by Zhang \& Cheng (1997). According to their model, for a given $\gamma$-ray pulsar with period, magnetic field and inclination angle, the fractional size of the outer gap, $f(r, P, B)$, is a function of $P, B$ and radial distance $r$ (see Zhang et al. 2004 in detail). The fractional size reaches a minimum at the radius $\left(r_{\text {in }}\right)$ of the inner boundary, and then increases with radius for a given pulsar. In order to explain the average properties of high-energy photon emission from the outer gap, we assume that high-energy emission at an average radius $\langle r\rangle$ represents the typical emission of high-energy photons from a pulsar. The average radius is given by

$\langle r\rangle=\frac{\int_{r_{\text {min }}}^{r_{\text {max }}} f(r, \alpha) r \mathrm{~d} r}{\int_{r_{\text {in }}}^{r_{\text {max }}} f(r, \alpha) \mathrm{d} r}$,

where $r_{\max }=\min \left(r_{c}, r_{b}\right)$. The average gap size, $f(\langle r\rangle, P, B)$, is generally a function of $P, B$ and $\alpha$ because $r_{c}$ is a function of $P$ and $\alpha$, and $r_{b}$ are function of $P, B$ and $\alpha$.

The derived outer gap size $f(\langle r\rangle(\alpha), P, B)$ is not only a function of period $(P)$ and magnetic field $(B)$ of the neutron star, but also a function of the average radial distance to the neutron star $\langle r\rangle$, which depends on the magnetic inclination angle $(\alpha)$. We use this new outer gap model to study the $\gamma$-ray luminosity of pulsars, which is given by

$L_{\gamma} \approx f^{3}(\langle r\rangle, P, B) L_{\mathrm{sd}}$,

where $L_{\mathrm{sd}}$ is the pulsar spin-down power. Generally, the inclination angles for the pulsars are not well known, so we consider the changes of the fractional size with the inclination angle. Let us define the following function:

$f(\langle r\rangle, P, B)=\eta(\alpha, P, B) f_{o}(P, B)$, where $f_{o}(P, B) \approx 5.5 P^{26 / 21} B_{12}^{-4 / 7}$ is the fractional size of outer gap, by ignoring the effect of inclination angle (Zhang \& Cheng 1997). So the function $\eta(\alpha, P, B)$ contains the effect of inclination angle, which can be expressed as (Zhang et al. 2004)

$\eta(\alpha, P, B) \approx \eta_{1} \times \eta_{2} \times \eta_{3}$,

where

$\eta_{1}=\left[\frac{2 \sqrt{1-\frac{3}{4} \frac{\langle r\rangle}{R_{L}} a(\alpha)}}{\sqrt{1-\frac{3}{4} \frac{\langle r\rangle}{R_{L}} a(\alpha)}+\sqrt{1-\frac{\langle r\rangle}{R_{L}} a(\alpha)}}\right]^{4 / 7}$,
$\eta_{2}=\left[\frac{4}{3} \frac{\left(1-\frac{3}{4} \frac{\langle r\rangle}{R_{L}} a(\alpha)\right)^{3 / 2}}{a^{3 / 2}(\alpha)\left(1-\frac{1}{2} \frac{\langle r\rangle}{R_{L}} a(\alpha)\right) \cos \alpha}\right]^{1 / 7}$,

and

$\eta_{3}=\frac{\left(\ln \frac{\langle r\rangle}{R}\right)^{\frac{1}{21}}\left(\frac{\langle r\rangle}{R_{L}}\right)^{\frac{13}{14}}}{\left[1-\frac{3\langle r\rangle a(\alpha)}{4 R_{L}}\right]^{\frac{3}{14}}}$.

In the above expression, $a(\alpha)=\sin ^{2}\left(\theta_{c}-\alpha\right) \sin \theta_{c}$, and $\theta_{c}$ is the polar angle for the oblique magnetic dipole rotator, in which the last open field line is a tangent to the light cylinder given by (Kapoor \& Shukre 1998)

$\tan \theta_{c}=-\frac{3}{4 \tan \alpha}\left(1+\left(1+8 \tan ^{2} \alpha / 9\right)^{1 / 2}\right)$.

Further, we can estimate the average energy flux of each $\gamma$-ray pulsar. Assuming the pulsar distance is $d=\left(d_{\mathrm{kpc}} / 1 \mathrm{kpc}\right)$ and the solid angle of $\gamma$-ray beaming is $\Delta \Omega$, then the average energy flux can be approximated as

$S_{\gamma}\left(E_{\gamma}>E_{1}\right) \approx 4.3 \times 10^{-11} \frac{\eta^{3} B_{12}^{2} P^{-4}}{\Delta \Omega d_{\mathrm{kpc}}} \quad \operatorname{erg~cm}^{-2} \mathrm{~s}^{-1}$,

where $L_{\mathrm{sd}} \approx 3.85 \times 10^{31} B_{12}^{2} P^{-4} \mathrm{erg} / \mathrm{s}$ has been used. Obviously, the average $\gamma$-ray flux depends on the magnetic inclination angle. In Eq. (9), the solid angle of $\gamma$-ray beaming relates to the model, and according to our outer gap model (Zhang et al. 2000), we have

$\Delta \Omega \approx 2 \pi\left(\frac{\alpha}{90^{\circ}}\right)^{0.5}\left(\frac{1-0.5 f(\langle r\rangle, P, B)}{1+0.5 f(\langle r\rangle, P, B)}\right)$

where $\alpha$ is the inclination angle and $f(\langle r\rangle, P, B)$ is given by Eq. (3). In our following simulations, we will use Eq. (10) to describe approximately the solid angle of $\gamma$-ray beaming.

\section{Monte Carlo simulation of $\gamma$-ray pulsars}

We simulate the $\gamma$-ray luminosity and spatial evolution of pulsars with ages of $2 \times 10^{7} \mathrm{yr}$ in the Galaxy and those with ages of $5 \times 10^{6} \mathrm{yr}$ in Gould belt using a Monte Carlo method. The basic procedure of the Monte Carlo method and the evolution of pulsar parameters are described in our previous works (Cheng \& Zhang 1998; Zhang \& Cheng 1999; Zhang et al. 2000, 2004; Cheng et al. 2004). We have made two improvements in our Monte Carlo simulation: (i) Parkes $70 \mathrm{~cm}$ survey and Princeton 
NRAO survey data are used and (ii) the distance model of Cordes \& Lazio (2002) is used to estimate the dispersion measure of the simulated pulsars. Here, we mainly describe the assumptions for generating the $\gamma$-ray pulsars in the Galaxy as well as in the Gould belt:

1 The pulsars are born at a rate $\left(\dot{N}_{\mathrm{NS}} \sim 1\right.$ per century) in the Galaxy, and at a rate of $\sim 20 \mathrm{Myr}^{-1}$ in the Gould belt (Grenier 2000). The initial period is chosen to be $P_{0}=10 \mathrm{~ms}$.

2 The initial position for each pulsar in the Galaxy is estimated from the distributions $\rho_{z}(z)=\left(1 / z_{\exp }\right) \exp \left(-|z| / z_{\exp }\right)$ and $\rho_{R}(R)=\left(a_{R} / R_{\text {exp }}^{2}\right) R \exp \left(-R / R_{\text {exp }}\right)$, where $z$ is the distance from the Galactic plane, $R$ is the distance from the Galactic center, $z_{\exp }=75 \mathrm{pc}, a_{R}=\left[1-e^{-R_{\max } / R_{\exp }}(1+\right.$ $\left.\left.R_{\max } / R_{\exp }\right)\right]^{-1}, R_{\exp }=4.5 \mathrm{kpc}$ and $R_{\max }=20 \mathrm{kpc}$ (Paczynski 1990; Sturner \& Dermer 1996). The Gould belt has an ellipsoidal shaped ring with semi-major and minor axes equal to $500 \mathrm{pc}$ and $340 \mathrm{pc}$ respectively. The Sun is displaced from the center of the Gould belt about $200 \mathrm{pc}$ towards $l=130^{\circ}$ (Guillout et al. 1998). The initial position of each pulsar is assumed to be uniformly inside the Gould belt.

3 The inclination angle ( $\alpha$ ) of each pulsar is chosen randomly from a uniform distribution (Biggs 1990).

4 The initial magnetic fields are distributed as a Gaussian in $\log B$ with a mean value of 12.5 and a dispersion of 0.3 . The majority of $\gamma$-ray pulsars are younger than 3 million years old and the field does not decay in $10 \mathrm{Myr}$ (Bhattacharya et al. 1992). So we ignore any field decay for these rotation-powered pulsars.

5 The initial velocity of each pulsar is the vector sum of the circular rotation velocity at the birth location and random velocity from the supernova explosion (Paczynski 1990). The circular velocity is determined by Galactic gravitational potential and the random velocity is distributed as a Maxwellian distribution with a dispersion of three dimensional velocity $=\sqrt{3} \times 100 \mathrm{~km} \mathrm{~s}^{-1}$ (Lorimer et al. 1997).

At time $t$, the period at time $t$ is given by $P(t)=\left(P_{0}+1.95 \times\right.$ $\left.10^{-39} B^{2} t\right)^{1 / 2}$. The pulsar position at time $t$ is determined following its motion in the Galactic gravitational potential. Using the equations given by Paczynski (1990) for given initial velocity, the orbit integrations are performed by using the 4th order Runge Kutta method with a variable time step on the variables $R, V_{R}, z, V_{Z}$ and $\phi$ (Press et al. 1992). Then the sky position and the distance of the simulated pulsar can be calculated.

In order to take radio selection effects into account, we simulate the Parkes $70 \mathrm{~cm}$ survey and Princeton NRAO survey phase I and II (for example, Manchester et al. 1996; Fan et al. 2001); the principal survey parameters are listed in Table 1 of Fan et al. (2001). The sky temperature is obtained using the program from ATNF and scaled to observing frequencies using a -2.6 power law of frequency dependence (Johnston et al. 1992). In our simulations, a pulsar that satisfies $L_{400} / d^{2} \geq S_{\text {min }}$ is considered to be a radio-detectable pulsar, where $L_{400}$ is the radio luminosity at $400 \mathrm{MHz}$ in units of $\mathrm{mJy} \mathrm{kpc}^{2}, d$ is the distance to the pulsar in units of kpc, and $S_{\min }$ is the survey sensitivity. $L_{400}$ of each model pulsar is estimated from the following distribution $\rho=0.5 \lambda^{2} \exp (-\lambda)$, where $\rho=$ $3.6\left(\log \left(L_{400} /\left\langle L_{400}\right\rangle\right)+1.8\right)$, and $\left.\left.\log \right\rangle L_{400}\right\rangle=(2 / 3) \log B_{12}+$ $(4 / 3) \log P+1.63$ (Narayan \& Ostriker 1990). We use the model of Cordes \& Lazio (2002) to calculate the dispersion measure. The radio beaming fraction can be expressed as (Emmering \& Chevalier 1989) $f_{r}(\omega)=(1-\cos \omega)+(\pi / 2-\omega) \sin \omega$, where $\omega=6.2 \times P^{-1 / 2}$ (e.g. Biggs 1990) is the half-angle of the radio emission cone. Then, following Emmering \& Chevalier (1989), a sample pulsar with a given period $P$ is chosen in one out of $f_{r}(P)^{-1}$ cases using the Monte Carlo method.

For the $\gamma$-ray threshold which varies over the sky, if $S^{\text {thr }}$ represents the $\gamma$-ray sensitivity, then a $\gamma$-ray pulsar is detectable when its energy flux is greater than $S^{\text {thr }}$ for a given detection instrument. For EGRET, we use the values given by Gonthier et al. (2002) which are

$S_{\gamma}\left(>E_{0}\right) \geq \begin{cases}1.2 \times 10^{-10} \mathrm{erg} \mathrm{cm}^{-2} \mathrm{~s}^{-1} & |b|<10^{\circ} \\ 7.0 \times 10^{-11} \mathrm{erg} \mathrm{cm}^{-2} \mathrm{~s}^{-1} & |b|>10^{\circ}\end{cases}$

where $E_{0}=100 \mathrm{MeV}$. For GLAST sensitivity, we use the values given by Gonthier et al. (2004), which are

$S_{\gamma}\left(>E_{0}\right) \geq\left\{\begin{array}{l}3.7 \times 10^{-12} \mathrm{erg} \mathrm{cm}^{-2} \mathrm{~s}^{-1}|b|<10^{\circ} \\ 1.5 \times 10^{-12} \mathrm{erg} \mathrm{cm}^{-2} \mathrm{~s}^{-1}|b|>10^{\circ}\end{array}\right.$

\section{Simulation results}

We simulated $\gamma$-ray pulsars with ages of $\leq 2 \times 10^{7}$ yr produced in the Galaxy and those with ages of $5 \times 10^{6} \mathrm{yr}$ in the Gould belt. In order to make a statistical analysis, we use the seed number of $6 \times 10^{6}$ in our simulations for the pulsars in Galaxy and seed number of $1.2 \times 10^{4}$ for the pulsars in Gould belt (it should be noted that the seed numbers used are greater than those estimated from the assumed neutron star birth rate by a factor of 30). To investigate the different properties of radio loud and radio quiet $\gamma$-ray pulsars, we produced three samples of $\gamma$-ray pulsars which contain all, radio quiet and radio loud $\gamma$-ray pulsars; all of those $\gamma$-ray pulsar have $f(\langle r\rangle, P, B) \leq 1$. Further we can consider $\gamma$-ray luminosity properties of $\gamma$-ray pulsars for different $\gamma$-ray sensitivities (say EGRET and GLAST).

We consider at first the spatial distributions of $\gamma$-ray pulsars using the EGRET sensitivity. In Figs. 1 and 2, we show the $\gamma$-ray pulsars simulated in the Galaxy and Gould belt respectively. It can be seen that there are different spatial distributions for the $\gamma$-ray pulsars in the Galaxy and in the Gould belt; the former has a tendency to concentrate on the galactic plane, but the latter is spatially relatively uniform. In our simulations, although pulsars produced in the Gould belt are fewer than those produced in the Galaxy, the ratio of $\gamma$-ray pulsars in the Gould belt to those in the Galaxy depends on the detector sensitivity. For example, the ratio is $\sim 51 \%$ for the EGRET threshold, but only 2.5\% for the GLAST threshold.

We are interested in the relation between the $\gamma$-ray luminosity and pulsar spin-down. We assume that $L_{\gamma} \propto L_{\text {sd }}^{\delta}$, where $\delta$ is a parameter which may be different for different $\gamma$-ray pulsar populations (say radio-quiet and radio loud $\gamma$-ray pulsars). In our simulations of the $\gamma$-ray pulsars produced in the Galaxy, in which the EGRET sensitivity is used, we find the following best fits

$\log L_{\gamma}=19.52+0.41 \log L_{\mathrm{sd}}$ 


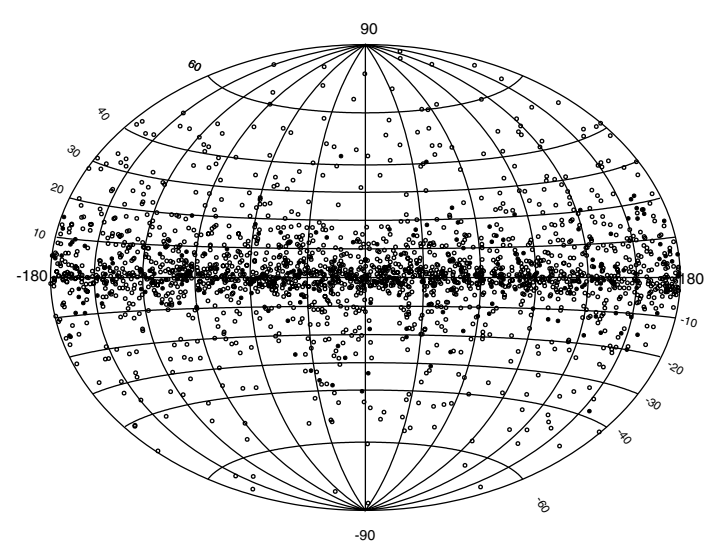

Fig. 1. Spatial distribution of the simulated $\gamma$-ray pulsars with ages of $\leq 2 \times 10^{7} \mathrm{yr}$ in the Galaxy. The birth rate of neutron stars is assumed to be $1 / 100 \mathrm{yr}$ and the $\gamma$-ray sensitivity is the EGRET sensitivity. Open (solid) circles represent radio quite (radio loud) $\gamma$-ray pulsars.

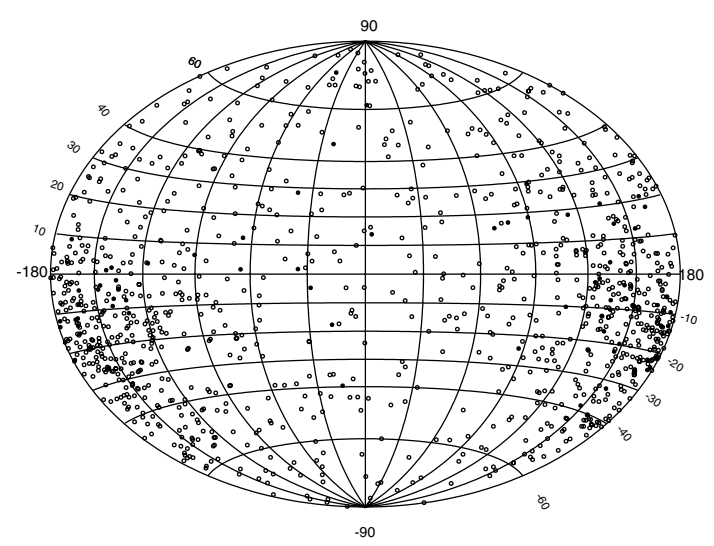

Fig. 2. Spatial distribution of the simulated $\gamma$-ray pulsars with ages of $\leq 5 \times 10^{6} \mathrm{yr}$ in the Gould belt. The birth rate of neutron stars is assumed to be $20 \mathrm{Myr}^{-1}$ and $\gamma$-ray sensitivity is the EGRET sensitivity. Open (solid) circles represent radio quite (radio loud) $\gamma$-ray pulsars.

for all $\gamma$-ray pulsars and radio-quiet $\gamma$-ray pulsars, and

$\log L_{\gamma}=19.96+0.39 \log L_{\text {sd }}$

for radio loud $\gamma$-ray pulsars. It can be seen that the relation between $L_{\gamma}$ and $L_{\mathrm{sd}}$ is similar for all and radio quiet $\gamma$-ray pulsars, the main reason being that radio-quiet $\gamma$-ray pulsars are dominant. The difference of the relations between radio-loud and radio-quiet $\gamma$-ray pulsars may be due to the radio selection effect. In Fig. 3, we show the relation between $L_{\gamma}$ and $L_{\text {sd }}$ for radio quiet and radio loud $\gamma$-ray pulsars in the Galaxy. Further, we consider the $\gamma$-ray pulsars produced in the Gould belt as follows

$\log L_{\gamma}=18.84+0.43 \log L_{\mathrm{sd}}$

for all $\gamma$-ray pulsars,

$\log L_{\gamma}=18.83+0.42 \log L_{\mathrm{sd}}$

for radio-quiet $\gamma$-ray pulsars, and

$\log L_{\gamma}=16.76+0.49 \log L_{\mathrm{sd}}$

for radio loud $\gamma$-ray pulsars. In Fig. 4, we show the relation between $L_{\gamma}$ and $L_{\mathrm{sd}}$ for radio quiet and radio loud $\gamma$-ray pulsars

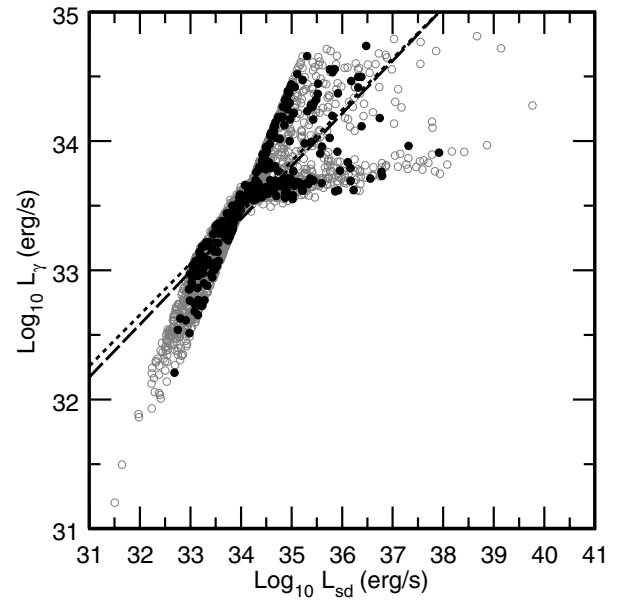

Fig. 3. Plot of $\gamma$-ray luminosity vs. spin-down luminosity for the simulated $\gamma$-ray pulsars in the Galaxy. The EGRET sensitivity has been used. Open and solid circles represent the radio quiet and radio loud $\gamma$-ray pulsars respectively. Long dashed line and short dashed line are corresponding best fits.

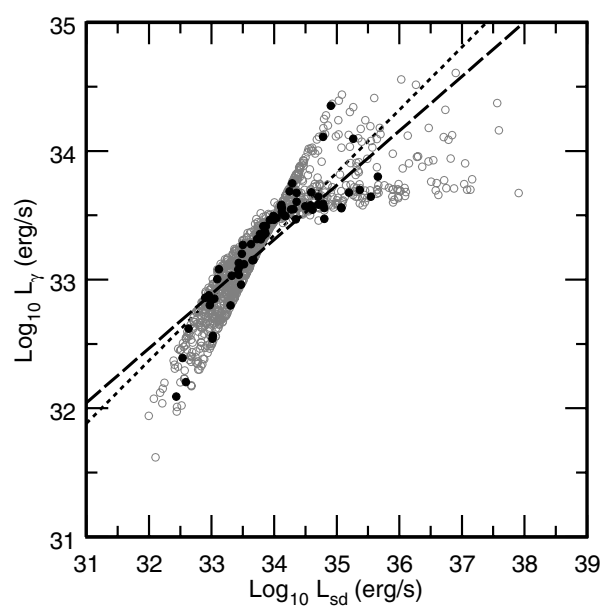

Fig. 4. Plot of $\gamma$-ray luminosity vs. spin-down luminosity for the simulated $\gamma$-ray pulsars in the Gould belt. The EGRET sensitivity has been used. Open and solid circles represent the radio quiet and radio loud $\gamma$-ray pulsars respectively. The long dashed line and short dashed line are corresponding best fits.

in the Gould belt. Compared to the $\gamma$-ray pulsars in the Galaxy, the $\gamma$-ray pulsars in the Gould belt have a steeper slope. Finally, the results for the $\gamma$-ray pulsars produced in both the Galaxy and Gould belt are

$\log L_{\gamma}=19.04+0.42 \log L_{\mathrm{sd}}$

for all $\gamma$-ray pulsars,

$\log L_{\gamma}=19.23+0.41 \log L_{\mathrm{sd}}$

for radio-quiet $\gamma$-ray pulsars, and

$\log L_{\gamma}=19.08+0.42 \log L_{\mathrm{sd}}$

for radio loud $\gamma$-ray pulsars. In Fig. 5, we show the relation between $L_{\gamma}$ and $L_{\mathrm{sd}}$ for radio quiet and radio loud $\gamma$-ray pulsars in both the Galaxy and the Gould belt. 


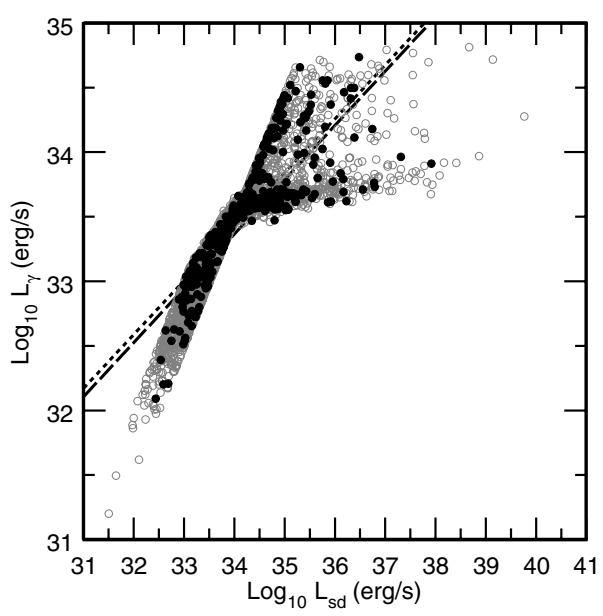

Fig. 5. Plot of $\gamma$-ray luminosity vs. spin-down luminosity for the simulated $\gamma$-ray pulsars in both the Galaxy and Gould belt. The EGRET sensitivity has been used. Open and solid circles represent the radio quiet and radio loud $\gamma$-ray pulsars respectively. the long dashed line and short dashed line are corresponding best fits.

We also find that the parameter $\delta$ varies with the $\gamma$-ray sensitivity. For example, using the GLAST sensitivity, the slope steepen and the relations between $L_{\gamma}$ and $L_{\mathrm{sd}}$ for the $\gamma$-ray pulsars produced in both the Galaxy and the Gould belt are as follows

$\log L_{\gamma}=18.31+0.44 \log L_{\mathrm{sd}}$

for all $\gamma$-ray pulsars,

$\log L_{\gamma}=18.25+0.44 \log L_{\mathrm{sd}}$

for radio-quiet $\gamma$-ray pulsars, and

$\log L_{\gamma}=19.64+0.40 \log L_{\mathrm{sd}}$

for radio loud $\gamma$-ray pulsars. Compared with the results for the EGRET threshold, on average, the values of $\delta$ increase slightly for the GLAST threshold.

The parameter $\delta$ also varies with the galactic latitude. In order to account for this, we divide the galactic latitude into 8 regions: $|b| \leq 5^{\circ},|b|=5^{\circ}-10^{\circ},|b|=10^{\circ}-20^{\circ},|b|=20^{\circ}-30^{\circ}$, $|b|=30^{\circ}-40^{\circ},|b|=40^{\circ}-50^{\circ},|b|=50^{\circ}-70^{\circ},|b|=70^{\circ}-90^{\circ}$. The results are shown in Fig. 6 . In this figure, the open boxes represent the results of all $\gamma$-ray pulsars for the EGRET threshold, the solid circles represent the results of all and radio loud $\gamma$-ray pulsars for the GLAST threshold. It can be seen that (i) there is a significant difference in the parameter $\delta$ between the $\gamma$-ray pulsars at the galactic plane $\left(|b| \leq 5^{\circ}\right)$ and those at high latitudes; and (ii) the values of $\delta$ increase with galactic latitude. How can we explain the above results? After estimating the ratio of mature $\left(>10^{6} \mathrm{yr}\right) \gamma$-ray pulsars to total $\gamma$-ray pulsars in each latitude region, we find that the ratios increase with the galactic latitude. We show the results in Fig. 8. It can be seen that the young $\gamma$-ray pulsars are dominant in the galactic plane, but the mature $\gamma$-ray pulsars will be dominant at high galactic latitudes. Therefore, we may conclude that mature $\gamma$-ray pulsars have different relations of $L_{\gamma}$ and $L_{\mathrm{sd}}$ than young $\gamma$-ray pulsars.

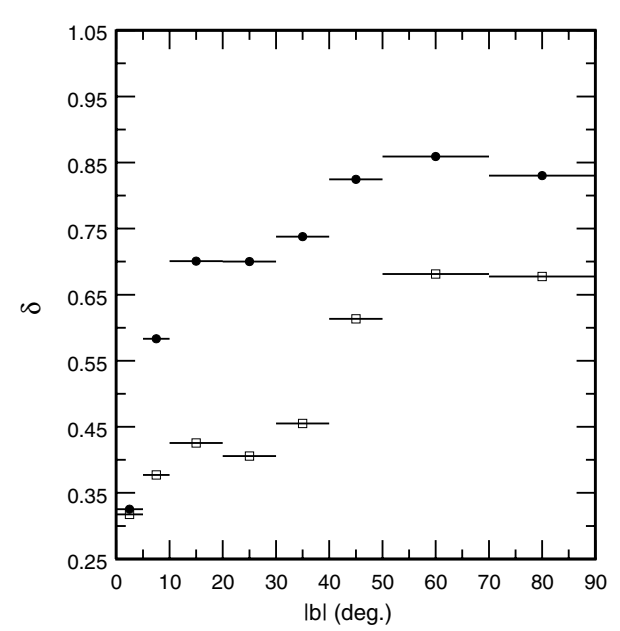

Fig. 6. Variation of parameter $\delta$ with galactic latitude. The parameter $\delta$ is defined as $L_{\gamma} \propto L_{\text {sd }}^{\delta}$. For the EGRET sensitivity, the results are shown by open boxes. For the GLAST sensitivity, solid boxes represent values of $\delta$ at different galactic latitude regions for all $\gamma$-ray pulsars.

\section{Discussion and conclusion}

We have studied the $\gamma$-ray luminosity properties of pulsars with ages of $\leq 2 \times 10^{7} \mathrm{yr}$ in the Galaxy as well as those with ages of $\leq 5 \times 10^{6} \mathrm{yr}$ in the Gould belt in the framework of the outer gap model proposed by Zhang et al. (2004) using a Monte Carlo method. The $\gamma$-ray pulsars produced in the Galaxy have a spatial distribution concentrated on the galactic plane (see Fig. 1), but the $\gamma$-ray pulsars produced in the Gould belt have a relatively uniform spatial distribution (see Fig. 2). Assuming that the relation between $\gamma$-ray luminosity and the spin down power satisfies $L_{\gamma} \propto L_{\text {sd }}^{\delta}$ with a parameter $\delta$, we statistically analyzed the dependence of $\gamma$-ray luminosity of the simulated $\gamma$-ray pulsars on both the galactic latitude $|b|$ and $\gamma$-ray detection sensitivity $S^{\text {thr }}$, i.e the dependence of parameter $\delta$ on both $|b|$ and $S^{\text {thr }}$. For the EGRET sensitivity, we have given the relations between $L_{\gamma}$ and $L_{\mathrm{sd}}$ for radio-quiet and radio-loud $\gamma$-ray pulsars produced in the Galaxy, Gould belt, and both the Galaxy and Gould belt, respectively (see Figs. 3-5). One feature is that $\delta(\sim 0.49)$ for radio-loud $\gamma$-ray pulsars in the Gould belt is greater than that $(\sim 0.42)$ for radio-quiet $\gamma$-ray pulsars; this case is different to that in the Galaxy. Our results further indicate that $\delta$ is a function of $|b|$ and $S^{\text {thr }}$. For a given $S^{\text {thr }}, \delta$ increases with the galactic latitude $|b|$. For the EGRET threshold, $\gamma$-ray pulsars at $|b| \leq 5^{\circ}$ are dominated by young pulsars ( $\sim 90 \% \gamma$-ray pulsars have ages of less than $10^{6} \mathrm{yr}$ ), therefore the parameter $\delta$ has a minimum value. However, the ratio of mature $\gamma$-ray pulsars to total $\gamma$-ray pulsars above the region of $|b|>5^{\circ}$ increases rapidly. A similar case can be seen for the GLAST threshold, for example, where the value of $\delta$ at $|b|=5^{\circ}-10^{\circ}$ is greater than that at $|b| \leq 5^{\circ}$ by a factor of $\sim 2$, and mature $\gamma$-ray pulsars with ages of $>10^{6}$ yr occupy $\sim 66 \%$ of all $\gamma$-ray pulsars at this latitude region (it is $\sim 14 \%$ at $|b| \leq 5^{\circ}$ ). At high galactic latitudes with $|b|>10^{\circ}$, mature $\gamma$-ray pulsars are dominant (>90\%), and the $\delta$ varies smoothly with $|b|$. For a given galactic latitude region, $\delta$ increases with high $\gamma$-ray sensitivity, for example, the $\delta$ of all simulated $\gamma$-ray pulsars changes 


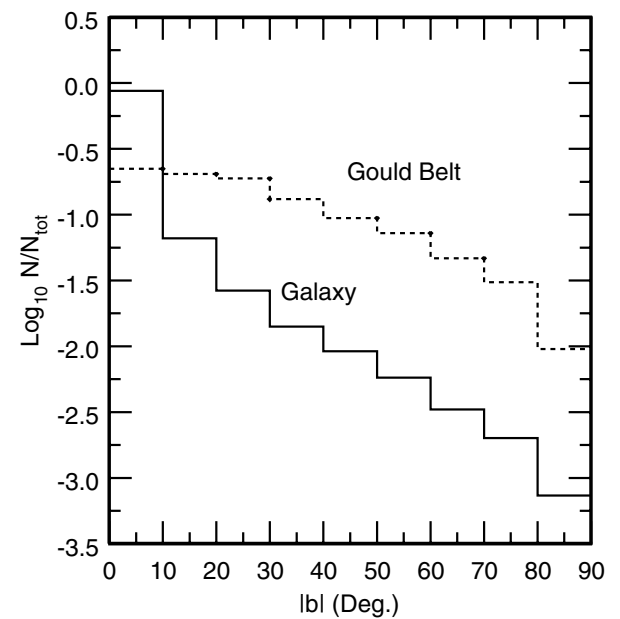

Fig. 7. Ratio of $\gamma$-ray pulsars at different galactic latitude regions to total $\gamma$-ray pulsars for the EGRET sensitivity. Solid line represents the ratio in the Galaxy and dashed line in the Gould belt.

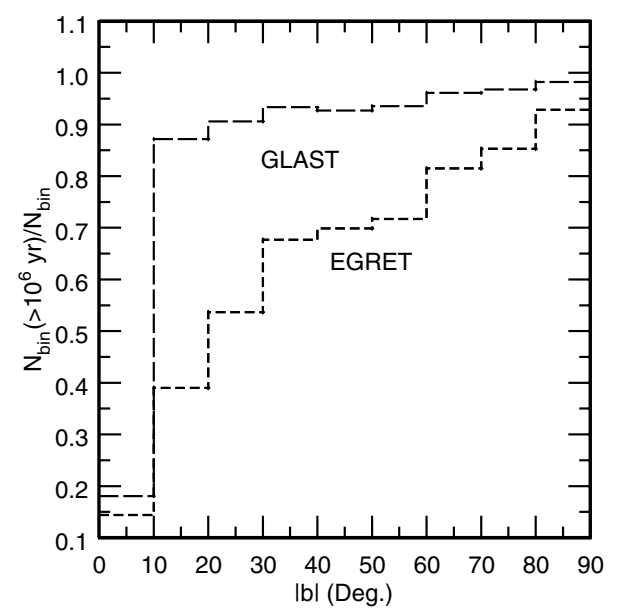

Fig. 8. Ratio of $\gamma$-ray pulsars with ages of $\leq \times 10^{6}$ yr to $\gamma$-ray pulsars at different galactic latitude regions for the EGRET (short dashed line) and the GLAST (long dashed line) sensitivities, respectively.

from $\sim 0.42$ for the EGRET sensitivity to $\sim 0.45$ for the GLAST sensitivity.

The main differences between the simulated $\gamma$-ray pulsars produced in the Gould belt and those produced in the Galaxy are as follows. At first, the spatial distribution of $\gamma$-ray pulsars produced in the Gould belt is flatter than that in the Galaxy; the ratio of $\gamma$-ray pulsars to total $\gamma$-ray pulsars for the GLAST sensitivity is shown in Fig. 7. It can be seen that the spatial distribution changes slowly for the $\gamma$-ray pulsars produced in the Gould belt(the ratio of maximum value to minimum value is $\sim 23$ ), but the $\gamma$-ray pulsars produced in the Galaxy mainly concentrate on the galactic plane $(\sim 90 \% \gamma$-ray pulsars are within the galactic latitude of $|b| \leq 10^{\circ}$ ). Secondly, the parameter $\delta$ varies smoothly with the galactic latitude for the $\gamma$-ray pulsars produced in the Gould belt, but has a abruptly jump from $|b| \leq 5^{\circ}$ to $|b|=5^{\circ}-10^{\circ}$ by a factor of $\sim 2$ for the $\gamma$-ray pulsars produced in the Galaxy.

We have used the new outer gap model proposed by Zhang et al. (2004) in the present work. The new model allows the outer gap to exist for a few million years old for appropriate combinations of $B, P$ and $\alpha$, thus old $\gamma$-ray pulsars can move up to high galactic latitude. Cheng et al. (2004) have used this model to study the $\gamma$-ray emission properties of $\gamma$-ray pulsars with ages of $\leq 10^{7} \mathrm{yr}$ in both the Galaxy and the Gould belt. They have shown that this model predicted very similar numbers of $\gamma$-ray pulsars as old models but many more high latitude $\gamma$-ray pulsars than old models (Cheng \& Zhang 1998; Zhang et al. 2000). Cheng et al. (2004) have obtained the spatial distribution, distance, period, age, magnetic field, inclination angle, photon flux distributions of the radio-loud and radioquiet $\gamma$-ray pulsars, and found out that the properties of $\gamma$-ray pulsars between $|b|<5^{\circ}$ and $|b|>5^{\circ}$ are very different: the high latitude $\gamma$-ray pulsars satisfy $L_{\gamma} \propto L_{\mathrm{sd}}^{0.6}$ but the galactic plane pulsars satisfy $L_{\gamma} \propto L_{\text {sd }}^{0.3}$ for the EGRET threshold. In our simulations, the values of $\delta$ for all, radio-quiet, and radio loud $\gamma$-ray pulsars produced in both the Galaxy and the Gould belt are $\sim 0.31, \sim 0.31$ and $\sim 0.33$ at $|b| \leq 5^{\circ}$, and $\sim 0.45$, $\sim 0.45$ and $\sim 0.45$ at $|b|>5^{\circ}$ for the EGRET threshold, respectively, consistent with those at the galactic plane given by Cheng et al. (2004), but there are some differences at high galactic latitude. The main reasons for these differences are that in this work (i) the $\gamma$-ray luminosity properties of $\gamma$-ray pulsars with ages of $<2 \times 10^{7} \mathrm{yr}$ in the Galaxy and those with ages of $5 \times 10^{6} \mathrm{yr}$ in the Gould belt are studied in detail after taking Parkes $70 \mathrm{~cm}$ survey and Princeton NRAO survey data and the distance model of Cordes \& Lazio (2002) into account; and (ii) the effect of $\gamma$-ray beaming is considered, according to our model (see Eq. (10)). Thus, the solid angle of $\gamma$-ray beaming can change by a factor of one order of magnitude for young and old pulsars, compared to the results obtained by assuming $\Delta \Omega=1$. The number of simulated $\gamma$-ray pulsars decreases when Eq. (10) is used and is $~ 50 \%$ of the pulsar number obtained by using $\Delta \Omega=1$.

Acknowledgements. We thank the anonymous referee for his/her very constructive comments. This work is partially supported by Hundred Talents Program of CAS and the National 973 Projection of China (NKBRSFG 19990754).

\section{References}

Bhattacharya, D., Wijers, R. A. M. J., Hartman, J. W., \& Verbunt, F. 1992, A\&A, 254, 198

Bhattacharya, D., Akyüz, A., Miyagi, T., Samimi, J., \& Zych, A. 2003, A\&A, 404, 163

Biggs, J. D. 1990, MNRAS, 245, 514

Camilo, F., Bell, J. F., Manchester, R. N., et al. 2001, ApJ, 557, L51

Cheng, K. S., Ho, C., \& Ruderman, M. A. 1986a, ApJ, 300, 500 (CHR I)

Cheng, K. S., Ho, C., \& Ruderman, M. A. 1986b, ApJ, 300, 522 (CHR II)

Cheng, K. S., \& Zhang, L. 1998, ApJ, 498, 327

Cheng, K. S., Zhang, L., Leung, P., \& Jiang, Z. J. 2004, ApJ, 608, 418

Cordes, J. M., \& Lazio, T. J. W. 2002 [arXiv: astro-ph/0202156]

Emmering, R. T., \& Chevalier, R. A. 1989, ApJ, 345, 931

Fan, G. L., Cheng, K. S., \& Manchester, R. N. 2001, ApJ, 557, 297

Gehrels, N., Macomb, D. J., \& Bertsch, D. L., et al. 2000, Nature, 404, 6776 
Gonthier, P. L., Ouellette, M. S., Berrier, J., O’Brien, S., \& Harding, A. K. 2002, ApJ, 565, 482

Gonthier, P. L., Van Guilder, R., \& Harding, A. K. 2004, ApJ, 604, 775

Grenier, I. 1997, Workshop on high energy cosmic neutrinos: origin, production and detection. June 2-3, 1997 - Marseille, France, Gamma-ray sources and diffuse emission above $100 \mathrm{MeV}$

Grenier, I. A. 2000, A\&A, 364, L93

Grenier, I. A. 2003, in Texas in Tuscany: XXI Symposium on Relativistic Astrophysics (Singapore: World Scientific) [arXiv: astro-ph/0303498]

Guillout, P., Sterzik, M. F., Schmitt, J. H. M. M., et al. 1998, A\&A, 337,113

Halpern, J. P., \& Ruderman, M. A. 1993, ApJ, 415, 286

Helfand, D. J. 1994, MNRAS, 267, 490

Harding, A. K., \& Zhang, B. 2001, ApJ, 548, L37

Hartman, R. C., Bertsch, D. L., Bloom, S. D., et al. 1999, ApJS, 123, 79

Hirotani, K., \& Shibata, S. 2002, ApJ, 564, 369

Johnston, S., Lyne, A. G., Manchester, R. N., et al. 1992, MNRAS, 255,401
Kaaret, P., \& Cottam, J. 1996, ApJ, 462, L35

Kramer, M., Bell, J. F., Manchester, R. N., et al. 2003, MNRAS, 341, 1299

Lorimer, D. R., Bailes, M., \& Harrison, P. A. 1997, MNRAS, 289, 592

Manchester, R. N., Lyne, A. G., D'Amico, N., et al. 1996, MNRAS, 279,1235

Montmerle, T. 1979, ApJ, 231, 95

Narayan, R., \& Ostriker, J. P. 1990, ApJ, 352, 222

Paczynski, B. 1990, ApJ, 348, 485

Press, W., Flannery, B., Teukolsky, S., \& Vetterling, W. 1992, Numerical Recipes: The Art of Scientific Computing 2nd ed. (Cambridge: Cambridge Univ. Press)

Romero, G. E., Benaglia, P., \& Torres, D. F. 1999, A\&A, 348, 868

Torres, D. F., Butt, Y. M., \& Camilo, F. 2001, ApJ, 560, L155

Yadigaroglu, I. A., \& Romani, R. W. 1995, ApJ, 449, 211

Yadigaroglu, I. A., \& Romani, R. W. 1997, ApJ, 476, 347

Zhang, L., \& Cheng, K. S. 1997, ApJ, 487, 370

Zhang, L., \& Cheng, K. S. 1998, A\&A, 335, 234

Zhang, L., \& Cheng, K. S. 1999, ApJ, 526, 327

Zhang, L., Zhang, Y. J., \& Cheng, K. S. 2000, A\&A, 357, 957

Zhang, L., Cheng, K. S., Jiang, Z. J., \& Leung, P. 2004, ApJ, 604, 317 